\title{
Effect of sex and seasons of the year on hematologic and serum biochemical variables of captive brown brocket deer (Mazama gouazoubira) $^{1}$
}

\author{
Clara M.S. Camargo² ${ }^{2}$ José M.B. Duarte ${ }^{3}$, José J. Fagliari², André M. Santana², \\ Kalina M.M.G. Simplício ${ }^{2}$ and Aureo E. Santana ${ }^{4 *}$
}

\begin{abstract}
Camargo C.M.S., Duarte J.M.B., Fagliari J.J., Santana A.M., Simplício K.M.M.G \& Santana A.E. 2013. Effect of sex and seasons of the year on hematologic and serum biochemical variables of captive brown brocket deer (Mazama gouazoubira). Pesquisa Veterinária Brasileira 33(11):1364-1370. Departamento de Clínica e Cirurgia, Faculdade de Ciências Agrárias e Veterinárias, Universidade Estadual Paulista, Júlio de Mesquita Filho, Unesp-Jaboticabal, Via de Acesso Prof. Paulo Donato Castellane s/n, Jaboticabal, SP 14884900, Brazil. E-mail:_santana@fcav.unesp.br

The Brown brocket deer (Mazama gouazoubira) is the most common free-living and captive deer in South America, especially in Brazil, and has great ecological and scientific significance. However, data on hematological and biochemical parameters in brown brocket deer are scarce. The goal of this study was to establish reference ranges for hematological and biochemical parameters of Mazama gouazoubira, comparing differences during the seasons of the year and between sex. Blood samples from ten adult healthy brown brocket deer ( 6 female and 4 male) were collected during daytime, monthly, during 12 months. The animals were maintained in individual stable, protected from noise and fed ad libitum with commercial ration and green fodder. For blood collection, animals were submitted to physical restrain for no longer than 2 minutes. The following parameters were determined: red blood cell count (RBC), haemoglobin concentration, packed cell volume (PCV), mean corpuscular volume (MCV), mean corpuscular haemoglobin (MCH), mean corpuscular haemoglobin concentration (MCHC), white blood cell count (WBC), platelet count, enzyme activity of alanine aminotransferase (ALT), aspartate aminotransferase (AST) and gamma-glutamyl transferase (GGT) and serum levels of alkaline phosphatase (ALP), creatine kinase (CK), total protein (TP), albumin, cholesterol, total calcium, ionic calcium, sodium, potassium, magnesium, triglycerides, creatinine and urea. Values were compared according to season and sex. RBC count, WBC count and MCV suggested seasonal influence. Haemoglobin concentration, PCV and MCV were influenced by sex. Serum concentration of total calcium, ionic calcium, sodium, potassium and magnesium were influenced by season. Serum magnesium was also influenced by sex. The blood parameters herein reported may be useful as reference values for diagnostic and prognostic purposes in captive brown-brocket deer.
\end{abstract}

INDEX TERMS: Brown brocket deer, hematology, Mazama gouazoubira, seasonality, serum biochemistry.

\footnotetext{
${ }^{1}$ Received on February 22, 2013.

Accepted for publication on October 22, 2013.

${ }^{2}$ Departamento de Clínica e Cirurgia Veterinária, Faculdade de Ciências Agrárias e Veterinárias (FCAV), Universidade Estadual Paulista (Unesp), Via de Acesso Prof. Paulo D. Castellane s/n, Jaboticabal, SP 14884-900, Brazil.

${ }^{3}$ Nucleo de Pesquisa e Conservação de Cervideos (NUPECCE), Departamento de Zootecnia, FCAV-Unesp, Jaboticabal, SP.

${ }^{4}$ Departamento de Clínica e Cirurgia Veterinária, FCAV-Unesp, Jaboticabal, SP. *Corresponding author: santana@fcav.unesp.br
}

RESUMO.- [Efeito do sexo e estações do ano sobre as variáveis hematológicas e bioquímico-séricas de veados-catingueiro (Mazama gouazoubira) criados em cativeiro.] 0 veado-catingueiro (Mazama gouazoubira) é o cervídeo de vida livre mais comum na América do Sul, especialmente no Brasil, e tem grande importância ecológica e científica. No entanto, dados sobre parâmetros hematológicos e bioquímicos nesta espécie animal são escassos. 0 
objetivo deste trabalho foi estabelecer valores de referência hematológica e bioquímica do Mazama gouazoubira, comparando diferenças entre as estações do ano e entre sexo. Amostras sanguíneas de dez veados-catingueiros (6 fêmeas e 4 machos), todos adultos hígidos, foram mensalmente colhidas pela manhã, durante 12 meses, e prontamente analisadas. Os animais foram mantidos em baias individual, protegidos de barulho e alimentados ad libitum com ração comercial e forragem verde. Para obtenção das amostras, os animais foram submetidos à contenção física por, no máximo, 2 minutos. Os seguintes parâmetros foram determinados: Contagem total de hemáceas, concentração de hemoglobina, volume globular (VG), volume corpuscular médio (VCM), hemoglobina corpuscular média (HCM), concentração de hemoglobina corpuscular média (CHCM), contagem total de leucócitos, contagem de plaquetas, atividade das enzimas alanina aminotransferase (ALT), aspartato aminotransferase (AST), gama glutamiltransferase (GGT) e concentrações séricas de fosfatase alcalina (FA), creatino quinase (CK), proteína total (PT), albumina, colesterol, cálcio total, cálcio iônico, sódio, potássio, magnésio, triglicérides, creatinina e uréia. Os valores foram comparados de acordo com o sexo e sazonalidade. A contagem total de hemáceas, de leucócitos e VCM sugeriu influência das estações do ano sobre estes parâmetros. A concentração de hemoglobina, VG e VCM foram influenciados pelo sexo. Concentrações séricas de cálcio total, cálcio iônico, sódio, potássio e magnésio também foram influenciados pelas estações do ano. 0 teor sérico de magnésio sofreu influência, ainda, do sexo. Os parâmetros sanguíneos obtidos e aqui reportados podem ser úteis como valores de referência nesta espécie para fins diagnóstico e prognóstico.

TERMOS DE INDEXAÇÃO: Veado-catingueiro, hematologia, Mazama gouazoubira, sazonalidade, bioquímica sérica.

\section{INTRODUCTION}

Brazil has an important biodiversity, although there is lack of information upon several parameters on native animal species. Eight cervid species, including Blastocerus dichotomus, Mazama nana, Mazama americana, Mazama gouazoubira, Mazama bororo, Mazama rufina, Odocoileus virginianus and Ozotoceros bezoarticus have been described in Brazil (Ministério do meio ambiente 2008). Mazama gouazoubira (Brown Brocket Deer) is characterized as a small cervid $(0.50 \mathrm{~m}$ tall and about $18 \mathrm{~kg}$ of body weight) with a wide variety of coat color, ranging from dark gray to reddish-brown (Duarte 1997). This species is endemic to neotropical regions, from southern Mexico to northern Argentina, and also has been registered in all Brazilian ecotopes (Pinder \& Leeuenberg 1997, Nowak 1999). For this reason, it assumes great ecological importance and represents a model for studies concerning reproduction, genetics and pathology of Cervidae.

Several studies involving disease in free-living and captive species of Cervidae have being performed during the last years (Szabó et al 2005, Tiemann et al. 2005, Machado et al. 2006, Szabó et al. 2006, Araújo Júnior et al. 2010, Lux Hoppe et al. 2010, Grazziotin et al. 2011, Sacchi et al. 2012), and, in addition, studies about variations in blood values related to disease (Cattey et al. 1990, Stoffregen et al. 2006, Severin et al. 2012) also have being performed, indicating the importance of knowledge of physiological parameters for blood and serum chemistry so that we can correctly evaluate an animal's state of health, including populations.

Studies about variations in blood values related to seasonality and nutrition (DeLiberto et al. 1989, Rawson et al. 1992, Barrozo et al. 2001, Santana et al. 2008, Gaspar-López et al. 2011, Das et al. 2012, Park et al. 2012), sex and age (Jain 1997, Santos 1999, Thorn 2000, Santana et al., 2008, Hoppe et al. 2010, Rafaj et al. 2011, Alhuay et al. 2011) and type of capture method and stress (Mautz et al. 1980, Fernández-Morán et al. 2000, Munerato et al. 2010, Hoppe et al. 2010) have being performed, but only a few with Mazama gouazoubira (Barrozo et al. 2001, Santana et al 2008, Munerato et al. 2010, Hoppe et al. 2010).

Taking into account the lack of information about hematology parameters and biochemical profile in Mazama gouazoubira, the aim of this study was to help establish reference ranges for both hematological and biochemical parameters for this species, comparing differences during the seasons of the year and between sex.

\section{MATERIALS AND METHODS}

Ten adult brown brocket deer (Mazama gouazoubira), four males and six females, were housed at the Deer Research and Conservation Center (NUPECCE) from São Paulo State University/Jaboticabal campus. Animals were maintained individually in stalls and kept away from people and automobile. They were fed ad libitum with a commercial ration for horses (Passeio, Purina®, Paulínia, SP 13140-000, Brazil), fresh alfafa, perennial soy-bean and mulberry branches. The experiment was accomplished from July 2003 to June 2004. According to data from the Weather Station in the Department of Exact Sciences, Unesp-Jaboticabal campus, the mean atmospheric temperature during winter, spring, summer and autumn through the experiment was respectively 20.6, 24.3, 23.7 and $19.9^{\circ} \mathrm{C}$. During the same period, the values for mean sunstroke were respectively, 260,2; 224.8, 218.1 and 207.1h, thus during summer and autumn there was less luminosity (mean value of $10.7 \mathrm{~h}$ ) and during winter and spring there was more luminosity (mean value of $13.3 \mathrm{~h}$ ).

Aiming to minimize stressful stimuli animals were physically restrained for no longer than 2 minutes for blood sampling. Blood was drawn from the jugular vein using tubes containing $10 \%$ ethylene diamine tetra acetic acid (EDTA) for blood count, and plain tubes for chemical analysis of the decanted serum. Samples were kept in isothermic box with ice and transported to the Laboratory of Veterinary Clinical Pathology from São Paulo State University/ Jaboticabal campus for analysis. Samples were monthly obtained during morning, through a twelve month period. RBC and WBC counts were performed manually in a Neubauer chamber. Platelet count and WBC differential count were obtained from smears fixed with methanol and stained with May-Grunwald and Giemsa solutions. PCV was determined by the microhaematocrit method in capillary tubes and centrifuged at $12,000 \mathrm{~g}$ for 5 minutes (Jain 1993). MCV was obtained by the microhematocrit method and haemoglobin concentration by the use of commercial kits (Labtest, Labtest Diagnostica, Lagoa Santa, MG, 33400-000, Brazil) and read in spectrophotometer (Labquest model BIO 2000, Labtest Diagnóstica, Lagoa Santa, MG 33400-000, Brazil). The hematimetric indices MCV, MCHC and MCH were calculated. 
Serum activities of the enzymes ALT and AST (Reitman-Frankel method), ALP (Roy modified method), CK (Tanzer and Gilvarg method), GGT (Szasz modified method) and serum levels of total protein (Biuret method), albumin (Bromocresol green method), triglycerides and cholesterol (Enzymatic Trinder method), total calcium (Labtest method), ionic calcium (Labtest method), ionic sodium (Labtest method), ionic potassium (Labtest method), urea (diacetilmonoxin modified method) and creatinine (Lustosa-Basques method) were determined by commercial kits (Labtest, Labtest Diagnostica, Lagoa Santa, MG 33400-000, Brazil) and read in spectrophotometer (Labquest model BIO 2000, Labtest Diagnóstica, Lagoa Santa, MG 33400000, Brazil).

Statistical analysis was performed using Statistical Analysis System (SAS). All data was expressed as mean \pm standard deviation. Means were compared by Student t-test. Means obtained in different seasons (spring: September to December; summer: December to March; autumn: March to June and winter: June to September) were compared by Tukey test $(\mathrm{p}<0.05)$.

\section{RESULTS}

Obtained values for RBC, platelet and WBC counts during different seasons of the year are listed in Table 1. It was observed that RBC count was higher during summer time and lower during the winter, although MVC turned to be lower during summer and higher in winter. WBC count, as MCV, were found to be higher on winter time. No statistical differences were found between seasons in other hematological parameters studied.
Reference intervals for hematologic variables arranged by gender are given in Table 2. It is shown that haemoglobin, PCV and MCV values were found to be higher in male deers. No other statistical differences were observed in hematological parameters between male and female.

Reference intervals from biochemical profile of captive Brown-brocket deer (Mazama gouazoubira) during different seasons of the year are shown on Table 3. Total calcium and magnesium concentrations were found to be higher during spring, although the former showed lower levels on autumn and the latter was lower during winter. Ionic calcium presented higher levels on spring time while the lowest levels were observed during winter. The highest levels of ionic sodium were observed on summer time while the lowest levels of ionic potassium were obtained during spring. No statistical differences were found between remaining biochemical parameters studied.

Table 4 shows reference intervals from biochemical profile arranged by gender. No statistical differences were observed between male and female except for magnesium levels which were higher on the former.

\section{DISCUSSION}

RBC count and MCV were influenced by seasons. RBC count means were found to be lower at winter and higher at summer, while the means of MCV were higher in winter and lower in summer. Similarly to MCV, WBC count was found to

Table 1. Means, standard deviation and minimum-maximum values of hemogram and plaquetogram in captive brown brocket deer (Mazama gouazoubira), during seasons of the year

\begin{tabular}{|c|c|c|c|c|}
\hline Parameters & Spring & Summer & Autumn & Winter \\
\hline $\mathrm{RBC}(\mathrm{x} 106 / \mu \mathrm{L})$ & $\begin{array}{l}13.6 \pm 1.93 b \\
(9.37-18.5)\end{array}$ & $\begin{array}{c}15.7 \pm 3.78 c \\
(11-24.2)\end{array}$ & $\begin{array}{l}15.4 \pm 1.27 c \\
(11.9-17.2)\end{array}$ & $\begin{array}{l}11.8 \pm 1.91 a \\
(8.85-14.8)\end{array}$ \\
\hline hemoglobin $(\mathrm{g} / \mathrm{dL})$ & $\begin{array}{l}15.2 \pm 1.62 a \\
(12.8-18.9)\end{array}$ & $\begin{array}{c}14.5 \pm 1.95 a \\
(11-19.2)\end{array}$ & $\begin{array}{l}15.2 \pm 1.39 a \\
(12.8-18.1)\end{array}$ & $\begin{array}{l}15.4 \pm 1.43 a \\
(12.6-18.9)\end{array}$ \\
\hline PCV (\%) & $\begin{array}{l}43 \pm 5.7 a \\
(35-56)\end{array}$ & $\begin{array}{c}40 \pm 4 a \\
(30-50)\end{array}$ & $\begin{array}{l}43 \pm 4.3 a \\
(35-52)\end{array}$ & $\begin{array}{l}42 \pm 4.7 a \\
(34-53)\end{array}$ \\
\hline MCV (fL) & $\begin{array}{c}32.3 \pm 5.51 b \\
(23.4-50)\end{array}$ & $\begin{array}{c}25.9 \pm 4.82 \mathrm{c} \\
(16-32)\end{array}$ & $\begin{array}{l}27.8 \pm 2.06 \mathrm{c} \\
(23.3-31.8)\end{array}$ & $\begin{array}{l}36.7 \pm 5.9 a \\
(27.4-47)\end{array}$ \\
\hline MCH (pg) & $\begin{array}{c}11.3 \pm 1.79 \mathrm{a} \\
(8.6-17.8)\end{array}$ & $\begin{array}{c}9.47 \pm 1.79 a \\
(5-12.2)\end{array}$ & $\begin{array}{c}9.89 \pm 0.64 a \\
(8.9-11.5)\end{array}$ & $\begin{array}{c}13.3 \pm 2.17 \mathrm{a} \\
(10-16.9)\end{array}$ \\
\hline $\mathrm{MCHC}(\%)$ & $\begin{array}{c}35.25 \pm 2.1 \mathrm{a} \\
(31-38)\end{array}$ & $\begin{array}{c}36.07 \pm 4.9 \mathrm{a} \\
(27-56)\end{array}$ & $\begin{array}{c}35.44 \pm 1.25 a \\
(33-38)\end{array}$ & $\begin{array}{c}36.48 \pm 1.35 \mathrm{a} \\
(35-40)\end{array}$ \\
\hline platelet $(\mathrm{x} 103 / \mu \mathrm{L})$ & $\begin{array}{l}326 \pm 42.8 \mathrm{a} \\
(259-410)\end{array}$ & $\begin{array}{l}345 \pm 47.1 \mathrm{a} \\
(280-419)\end{array}$ & $\begin{array}{l}331 \pm 42.9 a \\
(244-400)\end{array}$ & $\begin{array}{l}339 \pm 50.5 a \\
(270-450)\end{array}$ \\
\hline WBC $(\mathrm{x} 103 / \mu \mathrm{L})$ & $\begin{array}{c}4.41 \pm 1.2 \mathrm{~b} \\
(2-7.5)\end{array}$ & $\begin{array}{c}4.22 \pm 0.85 b \\
(2.5-6.4)\end{array}$ & $\begin{array}{c}3.61 \pm 1.39 \mathrm{~b} \\
(1.5-7.6)\end{array}$ & $\begin{array}{c}5.32 \pm 1.55 \mathrm{a} \\
(3-8.8)\end{array}$ \\
\hline NS $(x 103 / \mu \mathrm{L})$ & $\begin{array}{c}2.41 \pm 0.96 \mathrm{a} \\
(0.6-5.1)\end{array}$ & $\begin{array}{l}2.69 \pm 0.86 a \\
(1.68-5.12)\end{array}$ & $\begin{array}{c}2.22 \pm 0.92 \mathrm{a} \\
(0.882-4.48)\end{array}$ & $\begin{array}{l}3.35 \pm 1.37 a \\
(0.78-5.98)\end{array}$ \\
\hline $\mathrm{NB}(\mathrm{x} 103 / \mu \mathrm{L})$ & $\begin{array}{c}0.1 \pm 0.3 a \\
(0-1)\end{array}$ & $\begin{array}{c}0.3 \pm 0.6 \mathrm{a} \\
(0-2)\end{array}$ & $\begin{array}{c}0.2 \pm 0.4 \mathrm{a} \\
(0-1)\end{array}$ & $\begin{array}{c}0.2 \pm 0.4 \mathrm{a} \\
(0-1)\end{array}$ \\
\hline BAS $(x 103 / \mu L)$ & $\begin{array}{c}0.19 \pm 0.4 \mathrm{a} \\
(0-1)\end{array}$ & $\begin{array}{c}0.22 \pm 0.73 a \\
(0-3)\end{array}$ & $\begin{array}{c}0.07 \pm 0.38 \mathrm{a} \\
(0-2)\end{array}$ & $\begin{array}{c}0.07 \pm 0.26 a \\
\quad(0-1)\end{array}$ \\
\hline $\operatorname{EOS}(\times 103 / \mu \mathrm{L})$ & $\begin{array}{c}5.6 \pm 4.2 \mathrm{a} \\
(2-19)\end{array}$ & $\begin{array}{c}4.7 \pm 3.3 \mathrm{a} \\
(1-15)\end{array}$ & $\begin{array}{l}4 \pm 2.4 \mathrm{a} \\
(1-13)\end{array}$ & $\begin{array}{c}3.2 \pm 2.5 \mathrm{a} \\
(0-10)\end{array}$ \\
\hline MON $(x 103 / \mu L)$ & $\begin{array}{c}1.52 \pm 1.01 \mathrm{a} \\
(0-4)\end{array}$ & $\begin{array}{c}2.72 \pm 2.61 \mathrm{a} \\
(1-11)\end{array}$ & $\begin{array}{c}1.89 \pm 1.31 \mathrm{a} \\
(0-6)\end{array}$ & $\begin{array}{c}1.79 \pm 0.9 a \\
(0-3)\end{array}$ \\
\hline LINF $(x 103 / \mu \mathrm{L})$ & $\begin{array}{l}1.69 \pm 0.36 a \\
(1.16-2.32)\end{array}$ & $\begin{array}{l}1.34 \pm 0.55 a \\
(0.65-2.82)\end{array}$ & $\begin{array}{l}1.16 \pm 0.64 a \\
(0.13-3.13)\end{array}$ & $\begin{array}{l}1.66 \pm 0.53 a \\
(0.67-3.05)\end{array}$ \\
\hline
\end{tabular}

Means of a same line followed by identical minuscule letter do not differ among themselves by Tukey test $(\mathrm{P}>0.05)$. 
Table 2. Means, standard deviation and minimum-maximum values of hemogram and plaquetogram in male and female captive brown brocket deer (Mazama gouazoubira)

\begin{tabular}{lcc}
\hline Parameters & Female $(\mathrm{n}=6)$ & Male $(\mathrm{n}=4)$ \\
\hline RBC $(\mathrm{x} 106 / \mu \mathrm{L})$ & $13.76 \pm 2.97 \mathrm{a}$ & $14.63 \pm 1.53 \mathrm{a}$ \\
& $(8.85-24.19)$ & $(9.37-21.18)$ \\
hemoglobin $(\mathrm{g} / \mathrm{dL})$ & $14.34 \pm 1.29 \mathrm{a}$ & $16.38 \pm 1.32 \mathrm{~b}$ \\
& $(11-19.2)$ & $(13.1-18.9)$ \\
VG $(\%)$ & $39.76 \pm 3.63 \mathrm{a}$ & $46.33 \pm 4.39 \mathrm{~b}$ \\
& $(30-50)$ & $(35-56)$ \\
MCV (fL) & $29.86 \pm 6.18 \mathrm{a}$ & $32.48 \pm 6.47 \mathrm{~b}$ \\
& $(16-47)$ & $(19-50)$ \\
MCH (pg) & $10.81 \pm 2.31 \mathrm{a}$ & $11.49 \pm 2.17 \mathrm{a}$ \\
& $(5-16.9)$ & $(8-17.8)$ \\
MCHC $(\%)$ & $36.06 \pm 3.15 \mathrm{a}$ & $35.35 \pm 1.93 \mathrm{a}$ \\
& $(27-56)$ & $(31-42)$ \\
platelet $(\mathrm{x} 103 / \mu \mathrm{L})$ & $335.9 \pm 38.40 \mathrm{a}$ & $330.7 \pm 56.33 \mathrm{a}$ \\
& $(270-450)$ & $(244-420)$ \\
WBC $(\mathrm{x} 103 / \mu \mathrm{L})$ & $4.36 \pm 1.53 \mathrm{a}$ & $4.48 \pm 1.14 \mathrm{a}$ \\
& $(1.5-8.8)$ & $(1.9-7.1)$ \\
NS $(\mathrm{x} 103 / \mu \mathrm{L})$ & $2.7 \pm 0.52 \mathrm{a}$ & $2.64 \pm 0.44 \mathrm{a}$ \\
& $(0.78-3.53)$ & $1.61-3.62)$ \\
LINF $(\mathrm{x} 103 / \mu \mathrm{L})$ & $1.43 \pm 0.52 \mathrm{a}$ & $1.52 \pm 0.40 \mathrm{a}$ \\
& $(0.39-2.79)$ & $(0.71-2.68)$
\end{tabular}

Means of a same line followed by identical minuscule letter do not differ among themselves by Tukey test $(\mathrm{P}>0.05)$. be significantly higher during winter, decreasing during the year. These results suggest an increased erythroleukopoietic activity through this period, once young cells may increase MCV (Feldman et al. 2010) without raising RBC count. The increased erythropoiesis could improve leukocyte production (Feldman et al. 2010), which, in our opinion, may justify the greater number of leukocytes during winter.

Although there was no significant difference between RBC count of males and females, the mean values obtained for males were slightly higher than those for females. These results were similar to those found in previous reports with Mazama gouazoubira (Hoppe et al. 2010). Values of PCV, haemoglobin concentration and MCV were significantly higher in males, as has been previously accounted (Thrall 2004). Differently, Hoppe et al. (2010) found lower haemoglobin, PCV and MCV values in males of Mazama gouazoubira. Gaspar-López et al. (2011), studying male red deer (Cervus elaphus), reported that RBC, hematocrit and hemoglobulin increase during the mating season, being that in this period, high testosterone levels (Gaspar-López et al. 2010) may have a stimulant effect on erytropoiesis.

In this study, the ratio lymphocyte/neutrophil (L/N) mean value was 0.58 for males and 0.53 for females. These

Table 3. Means, standard deviation and minimum-maximum values of biochemical parameters in captive brown brocket deer (Mazama gouazoubira), during seasons of the year

\begin{tabular}{|c|c|c|c|c|}
\hline Parameters & Spring & Summer & Autumn & Winter \\
\hline $\operatorname{ALT}(\mathrm{U} / \mathrm{L})$ & $\begin{array}{l}15 \pm 3.9^{a} \\
(10-22)\end{array}$ & $\begin{array}{l}18 \pm 5.0^{\mathrm{a}} \\
(10-26)\end{array}$ & $\begin{array}{c}15 \pm 5^{\mathrm{a}} \\
(8.4-31)\end{array}$ & $\begin{array}{l}22 \pm 5.2^{\mathrm{a}} \\
(10-31)\end{array}$ \\
\hline AST (U/L) & $\begin{array}{l}79.2 \pm 22.5^{\mathrm{a}} \\
(41.9-131)\end{array}$ & $\begin{array}{l}83.5 \pm 50.6^{a} \\
(41.9-229)\end{array}$ & $\begin{array}{c}96.8 \pm 88^{\mathrm{a}} \\
(41.9-398)\end{array}$ & $\begin{array}{c}88.9 \pm 30.7^{\mathrm{a}} \\
(47-157)\end{array}$ \\
\hline $\operatorname{ALP}(U / L)$ & $\begin{array}{c}121 \pm 89.5^{\mathrm{a}} \\
(41-414)\end{array}$ & $\begin{array}{l}146 \pm 87.4^{a} \\
(49.8-440)\end{array}$ & $\begin{array}{l}124 \pm 97.3^{\mathrm{a}} \\
(49.8-440)\end{array}$ & $\begin{array}{l}141 \pm 102^{\mathrm{a}} \\
(58-514)\end{array}$ \\
\hline GGT (U/L) & $\begin{array}{l}55.76 \pm 13.96^{\mathrm{a}} \\
(38.18-95.44)\end{array}$ & $\begin{array}{l}58.20 \pm 17.80^{\mathrm{a}} \\
(31.81-101.8)\end{array}$ & $\begin{array}{l}68.10 \pm 31.07^{\mathrm{a}} \\
(38.18-190.9)\end{array}$ & $\begin{array}{l}51.48 \pm 16.7^{\mathrm{a}} \\
(31.8-89.08)\end{array}$ \\
\hline Total protein $(\mathrm{g} / \mathrm{dL})$ & $\begin{array}{l}8.42 \pm 0.69^{\mathrm{a}} \\
(7.36-9.69)\end{array}$ & $\begin{array}{c}8.21 \pm 0.85^{\mathrm{a}} \\
(7.10-10.95)\end{array}$ & $\begin{array}{c}7.90 \pm 0.90^{\mathrm{a}} \\
(6.39-10.32)\end{array}$ & $\begin{array}{l}8.22 \pm 0.68^{a} \\
(6.62-9.26)\end{array}$ \\
\hline Albumin $(\mathrm{g} / \mathrm{dL})$ & $\begin{array}{l}3.25 \pm 0.40^{\mathrm{a}} \\
(2.03-3.73)\end{array}$ & $\begin{array}{l}3.23 \pm 0.42^{\mathrm{a}} \\
(2.21-4.57)\end{array}$ & $\begin{array}{l}3.44 \pm 0.30^{\mathrm{a}} \\
(2.89-4.31)\end{array}$ & $\begin{array}{l}3.35 \pm 0.43^{\mathrm{a}} \\
(2.16-4.07)\end{array}$ \\
\hline Urea $(\mathrm{mg} / \mathrm{dL})$ & $\begin{array}{l}61.12 \pm 17.06^{\mathrm{a}} \\
(28.97-97.9)\end{array}$ & $\begin{array}{c}60.9 \pm 15.84^{\mathrm{a}} \\
(33-99.21)\end{array}$ & $\begin{array}{c}58.04 \pm 16.45^{\mathrm{a}} \\
(10-90.22)\end{array}$ & $\begin{array}{c}60.86 \pm 16.83^{a} \\
(35-120)\end{array}$ \\
\hline Creatinine (mg/dL) & $\begin{array}{l}1.74 \pm 0.24^{\mathrm{a}} \\
(1.28-2.28)\end{array}$ & $\begin{array}{l}1.74 \pm 0.21^{\mathrm{a}} \\
(1.32-2.33)\end{array}$ & $\begin{array}{l}1.67 \pm 0.19^{\mathrm{a}} \\
(1.07-2.06)\end{array}$ & $\begin{array}{l}1.58 \pm 0.16^{\mathrm{a}} \\
(1.25-2.02)\end{array}$ \\
\hline Creatine kinase $(\mathrm{U} / \mathrm{L})$ & $\begin{array}{l}204 \pm 111^{a} \\
(72-582)\end{array}$ & $\begin{array}{l}218 \pm 187^{a} \\
(72-896)\end{array}$ & $\begin{array}{c}144 \pm 77^{a} \\
(46.6-350)\end{array}$ & $\begin{array}{c}184 \pm 96.4^{a} \\
(72-485)\end{array}$ \\
\hline Triglycerides (mg/dL) & $\begin{array}{l}24.14 \pm 6.34^{\mathrm{a}} \\
(14.3-32.9)\end{array}$ & $\begin{array}{c}22.17 \pm 6.74^{a} \\
(11.4-37.9)\end{array}$ & $\begin{array}{l}21.13 \pm 6.06^{\mathrm{a}} \\
(12.1-31.5)\end{array}$ & $\begin{array}{l}19.18 \pm 3.98^{a} \\
(13.6-32.2)\end{array}$ \\
\hline Cholesterol (mg/dL) & $\begin{array}{l}74.53 \pm 13.42^{\mathrm{a}} \\
(51.18-108.9)\end{array}$ & $\begin{array}{l}72.34 \pm 14.73^{\mathrm{a}} \\
(50.59-100.5)\end{array}$ & $\begin{array}{l}80.18 \pm 15.65^{a} \\
(49.99-108.9)\end{array}$ & $\begin{array}{l}77.03 \pm 19.19^{a} \\
(47.02-115.4)\end{array}$ \\
\hline Total calcium (mg/dL) & $\begin{array}{c}8.78 \pm 0.70^{\mathrm{a}} \\
(7.76-10.29)\end{array}$ & $\begin{array}{l}8.45 \pm 0.89^{\mathrm{ab}} \\
(7.23-10.68)\end{array}$ & $\begin{array}{l}8.00 \pm 0.58^{\mathrm{b}} \\
(7.22-9.13)\end{array}$ & $\begin{array}{l}8.49 \pm 0.72^{\mathrm{ab}} \\
(7.08-10.21)\end{array}$ \\
\hline Magnesium (mg/dL) & $\begin{array}{l}3.05 \pm 0.92^{\mathrm{a}} \\
(2.36-5.06)\end{array}$ & $\begin{array}{l}2.87 \pm 0.57^{\mathrm{ab}} \\
(1.77-4.23)\end{array}$ & $\begin{array}{l}2.35 \pm 0.41^{\mathrm{bc}} \\
(1.25-2.93)\end{array}$ & $\begin{array}{l}2.00 \pm 0.62^{c} \\
(1.14-3.04)\end{array}$ \\
\hline Ionic calcium (mg/dL) & $\begin{array}{l}0.71 \pm 0.09^{\mathrm{a}} \\
(0.35-0.86)\end{array}$ & $\begin{array}{c}0.67 \pm 0.11^{\mathrm{ab}} \\
(0.5-0.86)\end{array}$ & $\begin{array}{l}0.66 \pm 0.10^{\mathrm{ab}} \\
(0.46-0.82)\end{array}$ & $\begin{array}{l}0.62 \pm 0.10^{\mathrm{b}} \\
(0.63-0.83)\end{array}$ \\
\hline Ionic sodium (mmol/L) & $\begin{array}{c}145.63 \pm 2.97^{\mathrm{b}} \\
(146-170)\end{array}$ & $\begin{array}{c}152.21 \pm 6.16^{\mathrm{a}} \\
(121-157)\end{array}$ & $\begin{array}{c}141.24 \pm 10.9^{\mathrm{b}} \\
(117-150)\end{array}$ & $\begin{array}{c}141.48 \pm 9.20^{b} \\
(140-150)\end{array}$ \\
\hline Ionic potassium (mmol/L) & $\begin{array}{c}4.83 \pm 0.56^{\mathrm{b}} \\
(4.1-7.2)\end{array}$ & $\begin{array}{c}5.46 \pm 0.89^{a} \\
(4.4-6.9)\end{array}$ & $\begin{array}{c}5.74 \pm 0.73^{a} \\
(4.5-7)\end{array}$ & $\begin{array}{l}5.66 \pm 0.69^{\mathrm{a}} \\
(4-6.1)\end{array}$ \\
\hline
\end{tabular}

Means of a same line followed by identical minuscule letter do not differ among themselves by Tukey test $(\mathrm{P}>0.05)$. 
Table 4. Means, standard deviation and minimum-maximum values of biochemical parameters in male and female captive brown brocket deer (Mazama gouazoubira)

\begin{tabular}{|c|c|c|}
\hline Parameters & Female $(n=6)$ & Male $(n=4)$ \\
\hline $\operatorname{ALT}(\mathrm{U} / \mathrm{L})$ & $\begin{array}{c}17.6 \pm 5.40^{\mathrm{a}} \\
(8.44-31.43)\end{array}$ & $\begin{array}{c}16.5 \pm 5.13^{a} \\
(8.44-26)\end{array}$ \\
\hline AST (U/L) & $\begin{array}{c}81.3 \pm 30.43^{a} \\
(41.9-183.3)\end{array}$ & $\begin{array}{c}97.71 \pm 79.70^{\mathrm{a}} \\
(41.9-398.1)\end{array}$ \\
\hline $\operatorname{ALP}(\mathrm{U} / \mathrm{L})$ & $\begin{array}{c}140.43 \pm 110.7^{\mathrm{a}} \\
(41-514)\end{array}$ & $\begin{array}{c}119 \pm 43.8^{a} \\
(58-232)\end{array}$ \\
\hline GGT (U/L) & $\begin{array}{c}58.86 \pm 24.84^{\mathrm{a}} \\
(31.81-190.9)\end{array}$ & $\begin{array}{c}62.65 \pm 17.89^{a} \\
(31.81-127.3)\end{array}$ \\
\hline Total protein $(\mathrm{g} / \mathrm{dL})$ & $\begin{array}{c}8.18 \pm 0.81^{\mathrm{a}} \\
(6.39-10.32)\end{array}$ & $\begin{array}{c}8.22 \pm 0.80^{\mathrm{a}} \\
(6.62-10.95)\end{array}$ \\
\hline Albumin $(\mathrm{g} / \mathrm{dL})$ & $\begin{array}{l}3.34 \pm 0.30^{\mathrm{a}} \\
(2.16-4.07)\end{array}$ & $\begin{array}{l}3.27 \pm 0.52^{\mathrm{a}} \\
(2.03-4.59)\end{array}$ \\
\hline Urea (mg/dL) & $\begin{array}{l}58.62 \pm 12^{a} \\
(10-81.56)\end{array}$ & $\begin{array}{c}63.25 \pm 22.21^{\mathrm{a}} \\
(29.57-120)\end{array}$ \\
\hline Creatinine $(\mathrm{mg} / \mathrm{dL})$ & $\begin{array}{l}1.66 \pm 0.16^{\mathrm{a}} \\
(1.25-2.17)\end{array}$ & $\begin{array}{l}1.75 \pm 0.27 a \\
(1.07-2.33)\end{array}$ \\
\hline Creatine kinase $(\mathrm{U} / \mathrm{L})$ & $\begin{array}{c}195 \pm 110^{a} \\
(69.96-582)\end{array}$ & $\begin{array}{c}171 \pm 150^{a} \\
(46.64-898)\end{array}$ \\
\hline Triglycerides $(\mathrm{mg} / \mathrm{dL})$ & $\begin{array}{l}22.09 \pm 6.52^{\mathrm{a}} \\
(10.7-37.9)\end{array}$ & $\begin{array}{c}22.00 \pm 5.96^{a} \\
(12.1-32.2)\end{array}$ \\
\hline Cholesterol (mg/dL) & $\begin{array}{l}75.92 \pm 15.79^{a} \\
(47.02-115.4)\end{array}$ & $\begin{array}{l}75.93 \pm 15.60^{a} \\
(48.8-105.3)\end{array}$ \\
\hline Total calcium (mg/dL) & $\begin{array}{c}8.47 \pm 0.83^{\mathrm{a}} \\
(7.23-10.68)\end{array}$ & $\begin{array}{l}8.37 \pm 0.68^{a} \\
(7.08-9.92)\end{array}$ \\
\hline Magnesium (mg/dL) & $\begin{array}{l}2.39 \pm 0.48^{a} \\
(1.14-3.49)\end{array}$ & $\begin{array}{l}2.88 \pm 0.88^{b} \\
(1.17-5.06)\end{array}$ \\
\hline Ionic calcium $(\mathrm{mg} / \mathrm{dL})$ & $\begin{array}{c}0.66^{\mathrm{a}} \\
(0.46-0.86)\end{array}$ & $\begin{array}{c}0.62^{\mathrm{a}} \\
(0.35-0.79)\end{array}$ \\
\hline Ionic sodium $(\mathrm{mmol} / \mathrm{L})$ & $\begin{array}{c}145.55^{\mathrm{a}} \\
(120-170)\end{array}$ & $\begin{array}{c}144.67^{\mathrm{a}} \\
(117-159)\end{array}$ \\
\hline Ionic potassium $(\mathrm{mmol} / \mathrm{L})$ & $\begin{array}{c}5.6^{\mathrm{a}} \\
(4-7.2)\end{array}$ & $\begin{array}{c}5.4^{\mathrm{a}} \\
(4.1-7)\end{array}$ \\
\hline
\end{tabular}

Means of a same line followed by identical minuscule letter do not differ among themselves by Tukey test $(\mathrm{P}>0.05)$.

results were higher than reported by Hoppe et al. (2010) ( 0.41 for males and 0.49 for females) and Munerato et al. (2010) (0.41) in captive Mazama gouazoubira and by Feldman et al. (2010) in domestic ruminants (0.5). There are divergent studies concerning the $\mathrm{L} / \mathrm{N}$ ratio of different species: 0.83 for Odocoileus hemionus (Anderson et al. 1970), 1.0 (Hawkey \& Hart 1985) and 0.52 (Gupta et al. 2007) for Axis axis, 0.78 for Muntiacus muntjak (Gupta et al. 2007), 1.07 for Elaphurus davidianus (Hawkey \& Hart 1985), 0.93 for Cervus duvauceli (Hawkey \& Hart 1985), 0.63 for Rangifer tarandus (Feldman et al. 2010), 1.20 (Padilla et al. 2000) and 0.34 (Rafaj et al. 2011) for Cervus elaphus, 0.54 for Cervus elaphus canadensis (Park et al. 2012). Lymphocytes/ neutrophils ratio in healthy animals ranges between 2.00 and 3.00, being that stress can lead to lymphopaenia and neutrophilia resultant from rise on cortisol plasmatic concentration (Jones \& Allison 2007). Thereby, stress caused by physical and/or chemical immobilization could influence these parameters and perhaps that is the reason for such divergent values described in the literature.

Seasonal changes in the expression of feedback mechanisms that control food intake probably involve both neural and endocrine signals (Forbes 1997). Changes in hormone levels often reflect the physiological adjustments that animals make as the environment changes (Wingfield \& Kena- gy 1991). It is suggested that the putative action of hormones may be mediated indirectly through changes in liver or gut function (Rhind et al. 1998). Although no hormone profile was obtained in our study a significant variation in a series of minerals, which concentrations are directly influenced by food intake, was observed. The total calcium and magnesium concentrations were influenced by seasonality. Calcium concentrations were higher in spring and lower in autumn, while magnesium concentration was lower during winter but also higher during spring. Still, magnesium concentration was the only biochemical parameter found to be influenced by sex, with higher values in males. According to Kay (1979) and Loudon et al. (1989) many ruminants exhibit seasonal variation in food intake with high voluntary intakes during summer and low intakes in the winter. This variation is primarily controlled by photoperiod. In our study total calcium and magnesium concentrations were found to be higher in periods of the year in which there was more luminosity (spring) when compared to the lower magnesium concentrations obtained during winter.

The same kind of reasoning supports the results obtained for serum concentrations of ionic calcium, sodium and potassium which were all influenced by season. It is important to quote that glucocorticoids, which are known as stress hormones, mobilizes energy reserves needed to cope with environmental challenges (Sapolsky 1992). Balfanz et al. (2006) observed significant glucocorticoid metabolite level changes in the annual lifecycle of the red deer (Cervus elaphus) with the highest values in winter and lowest in summer. This could explain the results obtained in our study where there was a significant difference in ionic calcium concentration between spring and winter, with higher range values in spring as well as for sodium concentrations which was found to be significantly higher in summer than in other seasons. However, potassium concentration was found to be significantly lower in spring.

The mean values of AST in this study were similar to those found by Park et al. (2012) (Cervus elaphus Canadensis), Grupta et al. (2007) (Axis axis and Muntiacus muntjak) and Alhuay et al. (2011) (Odocoileus virginianus) and lower than those found by Severin et al. (2012) (Cervus elaphus) and Rafaj et al. (2011) (Cervus elaphus). The CK mean values were much lower than those found by Rafaj et al. (2011) (Cervus elaphus). However, although the mean values of AST and CK were lower than in some studies, there was a large variation in our study (from $41.9 \mathrm{U} / \mathrm{L}$ to $398.1 \mathrm{U} / \mathrm{L}$ for AST; and from $46.64 \mathrm{U} / \mathrm{L}$ to $898 \mathrm{U} / \mathrm{L}$ for $\mathrm{CK}$ ), both reflecting overloaded or skeletal muscle injury. It is well known that cervids are very susceptible to stress, therefore there are several reports about variations in CK concentrations in cervids (Audige 1992, English \& Lepherd 1981, Padilla et al. 2000, Hoppe et al. 2010, Rafaj et al. 2011). Even though blood collection was accomplished as brief as possible, not exceeding 2 minutes of restraining, we were not able to totally avoid stress at the moment of sampling. This probably explains the acute stress response reflected in elevated AST and CK levels in some animals.

Some studies have reported seasonal variations in blood constituent values which were attributed to tempe- 
rature, food availability or reproductive status of the animal (Miller et al. 1991, Wollkers et al. 1994). In our country, there is no dramatic variation in temperature in between seasons and during the research the same nutritional condition was kept throughout the experiment; the females presented estrus but did not copulate and males shed their antlers. Thus, the differences in biochemical values could be attributed to physiological variations within species.

In conclusion, the blood parameters herein reported may be useful as reference values for diagnosis and prognosis purposes in captive Mazama gouazoubira. However, to reduce data misinterpretation, haematological/biochemical methodology and the restraining method must always be considered.

Acknowledgments.- To Fundação de Amparo à Pesquisa do Estado de São Paulo (FAPESP/Brazil) for the financial support.

\section{REFERENCES}

Alhuay D.A., Olga L.E., Arnaldo A.S., Néstor F.P., Patrícia R.M. \& Gianmarco R.M. 2011. Liver biochemistry profile in white-tailed deer (Odocoileus virginianus) reared in captivity. Revta Invest. Vet. Perú. 22(2):138-143.

Anderson A.E., Medin D.E. \& Bowden D.C. 1970. Erythrocytes and leukocytes in a Colorado mule deer population. J. Wildl. Manage. 34:389406.

Audige L. 1992. Serum biochemical values of rusa deer (Cervus timorensis russa) in New Caledonia. Aust. Vet. J. 69:268-271.

Araújo Jr J.P., Nogueira M.F. \& Duarte J.M.B. 2010. Survey for foot-and-mouth disease in the endangered marsh deer (Blastocerus dichotomus) from marshlands of Parana River Basin, Brazil. J. Wildl Dis. 46:939-943.

Balfanz F., Beiglböck C.H., Huber S., Palme R. \& Arnold W. 2006. The influence of season, food intake and social rank on cortisol secretion in red deer (Cervus elaphus). European Association of Zoo and Wildlife Veterinarians, $6^{\text {th }}$ Scientific Meeting, Budapest, Hungary. (Abstract)

Barrozo L.A., Toniolo G.H., Duarte J.M.B., Pinho M.P. \& Oliveira J.A. 2001. Padrão anual de variação da testosterona sérica, volume testicular e aspectos seminais de veado-catingueiro (Mazama gouazoubira) em cativeiro. Revta Bras. Reprod. Anim. 25(2):210-211.

Catley A., Kock R.A., Hart M.G. \& Hawkey C.M. 1990. Haematology of clinically normal and sick captive reindeer (Rangifer tarandus). Vet. Rec. 126:239-241.

Das A., Katole S., Kumar A., Gupta S.P., Saini M. \& Swarup D. 2012. Feed consumption, nutrient utilization and serum metabolite profile of captive blackbucks (Antelope cervicapra) fed diets varying in crude protein content. J. Anim. Physiol. Anim. Nutr. 96:442-449.

Deliberto T.J., Pfister J.A., Demarais S. \& Vreede G.V. 1989. Seasonal changes in physiological parameters of white-tailed deer in Oklahoma. J. Wildl. Manage. 53:533-539.

Duarte J.M.B. 1997. Biologia e Conservação de Cervídeos Sul-Americanos: Blastocerus, Ozotocerus e Mazama. Funep, Jaboticabal, SP. 238p.

Duarte J.M.B. 2007. Artiodactyla: Cervidae (veado-catingueiro, veado-campeiro e cervo-do-pantanal), p.641-664. In: Cubas Z.S., Silva J.C.R. \& Catão-Dias J.C. (Eds), Tratado de Animais Selvagens: medicina veterinária. Roca, São Paulo, SP.

English A.W. \& Lepherd E.E. 1981. The haematology and serum biochemistry of wild fallow deer (Dama dama) in New South Wales. J. Wildl. Manage. 17:289-295.

Feldman B.F., Zinkl J.G. \& Jain N.C. 2010. Schalm's Veterinary Hematology. $5^{\text {th }}$ ed. Blackwell Publishing, Ames, Iowa. 1344p.

Fernández-Morán J., Palomeque J. \& Peinado V.I. 2000. Medetomidine/ tiletamine/zolazepam and xylazine/tiletamine/zolazepam combinations for immobilization of fallow deer (Cervus dama). J. Zoo Wildl. Med. 31:62-64.
Forbes J.M. 1997. Voluntary food intake and diet selection in farm animals. CAB International, Wallingford. 540p.

Gaspar-López E., Landete-Castillejos T., Estevez J.A., Ceacero F. \& Gallego L. 2010. Biometrics, testosterone, cortisol and antler growth cycle in Iberian red deer stags (Cervus elaphus hispanicus). Reprod. Domest. Anim. 45:243-249.

Gaspar-López E., Landete-Castillejos T., Estevez J.A., Ceacero F., Gallero L., García A.J. 2011. Seasonal variations in Red deer (Cervus elaphus) hematology related to antler growth and biometrics measurements. J. Exp. Zool. 315:242-249.

Grazziotin A.L., Duarte J.M.B., Szabó M.P.J., Santos A.P., Guimarães A.M.S. Mohamed A., Vieira R.F.C., Barros Filho I.R., Biondo A.W. \& Messick J.B. 2011. Prevalence and Molecular Characterization of Mycoplasma ovis in Selected Free-Ranging Brazilian Deer Populations. J. Wildl. Dis. 47:1005-1011.

Gupta A.R., Patra R.C., Sainiand M. \& Swarup D. 2007. Haematology and serum biochemistry of Chital (Axis axis) and Barking deer (Muntiacus muntjak) reared in semi-captivity. Vet. Res. Commun. 31:801-808.

Hawkey C.M. \& Hart M.G. 1985. Normal haematological values of axis deer (Axis axis), Père Davis's deer (Elaphurus davidianus) and barasingha (Cervus duvauceli). Res. Vet. Sci. 39:247-248.

Hoppe E.G.L., Schmidt E.M.S., Zanuzzo F.S., Duarte J.M.B. \& Nascimento A.A. 2010. Haematology of captive grey-brocket deer (Mazama gouazoubira) (Fischer, 1814) (Cervidae: Odocoileinae). Comp. Clin. Pathol. 19:29-32.

Jain N.C. 1993. Comparative hematologic features of some avian and mammalian species, p.54-71. In: Jain N.C. (Ed.), Essentials of Veterinary Hematology. Lea and Febiger, Philadelphia, Pennsylvania.

Jones M.L. \& Allison R.W. 2007. Evaluation of the ruminant complete blood cell count. Vet. Clin. North Am., Food Anim., Pract, 23:377- 402. Doi:10.1016/j.cvfa.2007.07.002

Kay R.N.B. 1979. Seasonal changes of appetite in deer and sheep. Agric. Res. Council Res. Rev. 5:13-15.

Loudon A.S.I., Milne J.A., Curlewis J.D. \& McNeilly A.S. 1989. A comparison of seasonal changes and patterns of growth, voluntary feed intake and reproduction in juvenile and adult re deer (Cervus elaphus) and Per David's deer (Elaphurus davidianus) hinds. J. Endocrinol. 122:733-745.

Lux Hoppe E.G., Tebaldi J.H. \& Nascimento A.A. 2010. Helminthological screening of free-ranging grey brocket deer Mazama gouazoubira Fischer, 1817 (Cervidae: Odocoileini) from Brazilian Pantanal wetlands, with considerations on Pygarginema verrucosa (Molin, 1860) Kadenatzii, 1948 (Spirocercidae: Ascaropsinae). Braz. J. Biol. 70:417-423.

Machado R.Z., Duarte J.M.B., Dagnone A.S. \& Szabó M.P.J. 2006. Detection of Ehrlichia chaffeensis in Brazilian Marsh Deer (Blastocerus dichotomus). Vet. Parasitol. 139:262-266.

Mautz W.W., Seal U.S. \& Boardman C.B. 1980. Blood serum analyses of chemically and physically restrained white-tailed deer. J. Wildl. Manage. 44:343-351.

Miller K.V., Knox W.M., Marchinton R.L. \& Ware G.O. 1991. Variations in the serum chemistry of captive female white-tailed deer. J. Sci. 49:95-100.

Ministério do Meio Ambiente 2008. Livro Vermelho da Fauna Brasileira Ameaçada de Extinção. Vol.1 e 2. Brasília, DF. 1420p. (In Portuguese)

Munerato M.S., Duarte J.M.B., Pereira G.T. \& Marques J.A. 2010. Effects of physical and chemical immobilization on hematologic and biochemical variables in captive brown brocket deer (Mazama gouazoubira) Vet. Clin. Pathol. 39(4):454-463.

Nowak R.M. 1999. Walker's Mammals of the World. Vol.2. $6^{\text {th }}$ ed. The Johns Hopkins University Press, Baltimore. 1936p.

Padilla S., Bouda J., Quiroz-Rocha G.F., Dávalos J.L. \& Sánchez A. 2000. Biochemical and haematological values in venous blood of captive red deer (Cervus elaphus) at high altitude. Acta.Vet. Brno 69:327-331.

Park J.H., Kim S.W., Do Y.J., Kim H., Ko Y.G., Yang B.S., Shin D. \& Cho Y.M. 2012. Spent mushroom substrate influences Elk (Cervus Elaphus Canadensis) hematological and serum biochemical parameters. Asian-Aust. J. Anim. Sci. 25:320-324.

Pinder L. \& Leeuenberg F. 1997. Veado-catingueiro (Mazama gouazoubira Fischer, 1814). In: Duarte J.M.B. (Ed.), Biologia e Conservação de 
Cervídeos Sul-americanos: Blastocerus, Ozotocerus e Mazama. Funep, Jaboticabal.

Rafaj R.B., Toncic J., Vickovic I. \& Sostaric B. 2011. Haematological and biochemical values of farmed red deer (Cervus elaphus). Vet. Arhiv 81:513523.

Rawson R.E., Delgiudice G.D., Dziuk H.E. \& Mech L.D. 1992. Energy metabolism and hematology of white-tailed deer fawns. J. Wildl. Manage. 28:91-94.

Rhind S.M., McMillen S.R., Duff E., Hirst D. \& Wright S. 1998. Seasonality of meal patterns and hormonal correlates in red deer. Physiol. Behav. 65(2):295-302.

Sacchi A.B.V., Duarte J.M.B., André M.R. \& Machado R.Z. 2012. Prevalence and molecular characterization of Anaplasmataceae agents in free-ranging Brazilian marsh deer (Blastocerus dichotomus). Comp. Immunol. Microb. 35(4):325-334.

Sapolsky R.M. 1992. Stress, the Aging Brain and the Mechanisms of Neuron Death. MIT Press, Cambridge. 429p.

Santana A.M., Fagliari J.J., Camargo C.M.S., Santana A.E., Duarte J.M.B. \& Silva P.R.L. 2008. Serum protein concentrations of captive brown brocket deer (Mazama gouazoubira) determined by means of agarosis and sodium dodecyl sulphate-polyacrylamide (SDS-PAGE) gel electrophoresis. Arq. Bras. Med. Vet. Zootec. 60(6):1560-1563.

Santos L.C. 1999. Laboratório Ambiental. Unioeste, Cascavel, SP.

Szabó M.P.J., Matushima E.R., Castro M.B., Santana D.A., Paula C.D. \& Duarte J.M.B. 2005. Hematology of free-living Marsh deer (Blastocerus dichotomus) from Southeast Brazil. J. Zoo Wildl. Med. 36(3):463-469.

Szabó M.P.J., Labruna M.B., Vogliotti A. \& Duarte J.M.B. 2006. Ticks (Acari: Ixodidae) on small red brocket deer (Mazama bororo Duarte) along deer trails in the Atlantic rain forest of Southeastern Brazil. Syst. Appl. Acarol. 11(1):41-45.

Severin K., Masek T., Janicki Z., Konjevic D., Slavica A., Marinculic A., Martinkovic F., Vengust G. \& Dzaja P. 2012. Liver enzymes and blood metabolites in a population of free-ranging red deer (Cervus elaphus) naturally infected with Fascioloides magna. J. Helminthol. 86:190196.

Stoffregen W.C., Alt D.P., Palmer M.V., Olsen S.C., Waters W.R. \& Stasko J.A. 2006. Identification of haemomycoplasma species in anemic reindeer (Rangifer tarandus). J. Wild. Dis. 42:249-258.

Szabó M.P.J., Matushima E.R., Castro M.B., Santana D.A., Paula C.D. \& Duarte J.M.B. 2005. Hematology of free-living Marsh deer (Blastocerus dichotomus) from Southeast Brazil. J. Zoo Wild. Med. 36:463-469.

Thorn C.E. 2000. Normal hematology of the deer, p.1179-1183. In: Feldman B.F., Zinkl J.G. \& Jain N.C. (Eds), Schalm's Veterinary Hematology. $5^{\text {th }}$ ed. Lippincott Williams and Wilkins, Philadelphia.

Thrall M.A. 2004. Veterinary Hematology and Clinical Chemistry. Lippincott Williams and Wilkins, Philadelphia. 618p.

Tiemann J.C.H., Rodrigues A.A.R., Souza S.L.P., Duarte J.M.B. \& Genari S.M. 2005. Occurrence of anti-Neospora caninum antibodies in Brazilian cervids kept in captivity. Vet. Parasitol. 129:341-343.

Wingfield J.C. \& Kenagy G.J. 1991. Natural regulation of reproductive cycles, p.181-241. In: Pang P.K.T. \& Schreibman M.P. (Eds), Vertebrate Endocrinology: fundamentals and biochemical implications. Vol.4. Part B. Academic Press, New York.

Wollkers H., Wensing T. \& Schonewille J.T. 1994. Effect of undernutrition on haematological and serum biochemical characteristics in red deer (Cervus elaphus). Can. J. Zool. 72:1291-1296. 